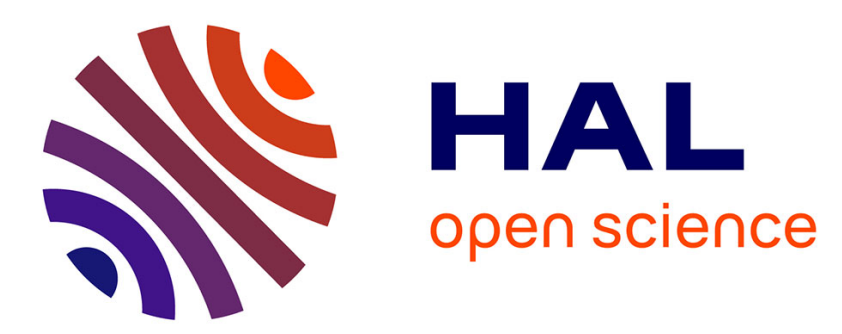

\title{
Description des courriels des patients pris en charge à l'Hôpital Européen Georges Pompidou, Paris, France
}

Vincent Looten, Antoine Neuraz, Nicolas Garcelon, Anita Burgun, Gilles Chatellier, Bastien Rance

\section{To cite this version:}

Vincent Looten, Antoine Neuraz, Nicolas Garcelon, Anita Burgun, Gilles Chatellier, et al.. Description des courriels des patients pris en charge à l'Hôpital Européen Georges Pompidou, Paris, France. EMOIS 2019, Mar 2019, Nancy, France. 67, pp.S104, 2019. hal-02069234

\section{HAL Id: hal-02069234 https://hal.science/hal-02069234}

Submitted on 15 Mar 2019

HAL is a multi-disciplinary open access archive for the deposit and dissemination of scientific research documents, whether they are published or not. The documents may come from teaching and research institutions in France or abroad, or from public or private research centers.
L'archive ouverte pluridisciplinaire HAL, est destinée au dépôt et à la diffusion de documents scientifiques de niveau recherche, publiés ou non, émanant des établissements d'enseignement et de recherche français ou étrangers, des laboratoires publics ou privés. 


\section{Description des courriels des patients pris en charge à l'Hôpital Européen Georges Pompidou, Paris, France}

\section{Looten ${ }^{1,2,3}$, A. Neuraz ${ }^{1,2,3}$, N. Garcelon ${ }^{2,4}$, A. Burgun ${ }^{1,2,3}$, G. CHATELLIER ${ }^{1,3}$, B. RANCE ${ }^{1,2,3}$}

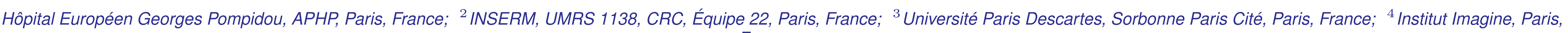
France;

\section{INTRODUCTION}

L'utilisation du courrier électronique pour régler des problèmes médicaux ou administratifs simples peut améliorer la qualité des soins. La demande des patients est forte, mais une revue Cochrane de 2014 [1] est demeurée inconclusive sur l'effet de la communication médecin/patient via courriel sur la performance des services de santé.Les problèmes de confidentialité ont déjà fait l'objet de publications. Notre objectif a été de décrire les emails des patients ainsi que leurs caractéristiques dans un hôpital universitaire

\section{MÉTHODE}

Tous les patients pris en charge à l'Hôpital Européen Georges Pompidou (HEGP) entre le 1/01/2000 et le 1/11/2018 ont été inclus. Les noms de domaines des courriels ont été extraits et comparés à une base publique. Les fournisseurs ont été identifiés par le service de recherche Whois. Les caractéristiques des patients avec ou sans courriel ont été comparées. Nous avons effectué un appariement sur l'année de premier contact avec l'hôpital pour prendre en compte l'évolution du taux de renseignement par l'administration au cours du temps.

\section{Discussion}

Le nombre de courriels disponibles est faible si on se réfère au taux d'utilisation national pour la santé (18,70\%)[2]; c'est un avantage sécuritaire (moindre exposition à une diffusion non contrôlée de données personnelles). Enfin, 8\% des patients ayant un courriel ouvrent à leur employeur un accès possible à leurs données médicales: l'éducation des patients et des médecins sur l'emploi de messageries sécurisées doit être renforcée.

\section{Tableau 3a : Description de la population appariée}

\begin{tabular}{|c|c|c|c|c|}
\hline & Population & Avec email & Sans email & $\boldsymbol{P}$ \\
\hline $\mathbf{N}$ & 82,008 & 41004 & 41004 & \\
\hline $\begin{array}{l}\text { Hommes, n } \\
(\%)\end{array}$ & $\begin{array}{c}39957 \\
(48.7 \%)\end{array}$ & $\begin{array}{c}20494 \\
(50.0 \%)\end{array}$ & $\begin{array}{c}19463 \\
(47.5 \%)\end{array}$ & $<0.001$ \\
\hline $\begin{array}{l}\text { Année de } \\
\text { naissance, } \\
\text { n (\%) }\end{array}$ & & & & \\
\hline Avant 1950 & $\begin{array}{c}20069 \\
(24.5 \%)\end{array}$ & $\begin{array}{c}10761 \\
(26.2 \%)\end{array}$ & $\begin{array}{c}9308 \\
(22.7 \%)\end{array}$ & \multirow{4}{*}{$<0.001$} \\
\hline 1950-1969 & $\begin{array}{c}27735 \\
(33.8 \%)\end{array}$ & $\begin{array}{c}11620 \\
(28.3 \%)\end{array}$ & $\begin{array}{c}16115 \\
(39.3 \%)\end{array}$ & \\
\hline 1970-1980 & $\begin{array}{c}26022 \\
(31.7 \%)\end{array}$ & $\begin{array}{c}13367 \\
(32.6 \%)\end{array}$ & $\begin{array}{c}12655 \\
(30.9 \%)\end{array}$ & \\
\hline Après 1989 & $\begin{array}{r}8182 \\
(10 \%)\end{array}$ & $\begin{array}{c}5256 \\
(12.8 \%)\end{array}$ & $\begin{array}{r}2926 \\
(7.2 \%)\end{array}$ & \\
\hline
\end{tabular}

Tableau 3b : Description de la population appariée

\begin{tabular}{|l|c|c|c|c|}
\hline $\mathbf{N}$ & Population & Avec email & Sans email & $P$ \\
\hline $\begin{array}{l}\text { Durée de } \\
\text { suivi, n (\%) }\end{array}$ & 82,008 & 41004 & 41004 & \\
\hline$<=3$ mois & 38743 & 27434 & 11309 & \\
& $(47.2 \%)$ & $(66.9 \%)$ & $(27.6 \%)$ & \\
\hline Entre 3 et 12 & 12591 & 5001 & 7590 & \\
mois & $(15.4 \%)$ & $(12.2 \%)$ & $(18.5 \%)$ & \\
Entre 12 et 24 & 7116 & 2492 & 4624 & \\
mois & $(8.7 \%)$ & $(6.1 \%)$ & $(11.3 \%)$ & \\
Entre 24 et 36 & 4336 & 1527 & 2809 & $<0.001$ \\
mois & $(5.3 \%)$ & $(3.7 \%)$ & $(6.9 \%)$ & \\
Entre 36 et 48 & 3106 & 1073 & 2033 & \\
mois & $(3.8 \%)$ & $(2.6 \%)$ & $(5 \%)$ & \\
Entre 48 et 60 & 2616 & 824 & 1792 & \\
mois & $(3.2 \%)$ & $(2.0 \%)$ & $(4.4 \%)$ & \\
>= 60 mois & 13500 & 2653 & 10847 & \\
& $(16.5 \%)$ & $(6.5 \%)$ & $(26.5 \%)$ &
\end{tabular}

\section{RÉsultats}

Durant la période d'étude, 971822 patients ont été pris en charge dont 41 $004(4,2 \%)$ avaient un courriel enregistré dans le système d'information $(9,5 \%$ avant $2005 ; 10,2 \%$ entre 2005 et $2010,80,3 \%$ après 2010$)$

\section{Tableau 1 : Description de la population}

\begin{tabular}{|l|c|c|c|c|}
\hline & $\begin{array}{c}\text { Population } \\
\text { totale }\end{array}$ & $\begin{array}{c}\text { Population } \\
\text { avec email } \\
\text { renseigné }\end{array}$ & $\begin{array}{c}\text { Population } \\
\text { après } \\
\text { appariement }\end{array}$ & $\begin{array}{c}\text { Population } \\
\text { exclus }\end{array}$ \\
\hline $\mathbf{N}$ & 971,822 & 41004 & 82008 & 889814 \\
\hline $\begin{array}{l}\text { Hommes, n } \\
\text { (\%) }\end{array}$ & 485737 & 19463 & 39957 & 445780 \\
Année de & $(50.0 \%)$ & $(47.5 \%)$ & $(48.7 \%)$ & $(50.1 \%)$ \\
naissance, n & & & & \\
(\%) & & & & \\
\hline Avant 1950 & 342676 & 9308 & 20069 & 322607 \\
& $(35.3 \%)$ & $(22.7 \%)$ & $(24.5 \%)$ & $(36.3 \%)$ \\
\hline $1950-1969$ & 282381 & 16115 & 27735 & 254646 \\
& $(29.1 \%)$ & $(39.3 \%)$ & $(33.8 \%)$ & $(28.6 \%)$ \\
\hline $1970-1989$ & 281390 & 12655 & 26022 & 255368 \\
& $(29.0 \%)$ & $(30.9 \%)$ & $(31.7 \%)$ & $(28.7 \%)$ \\
\hline Après 1990 & 65375 & 2926 & 8182 & 57193 \\
& $(6.7 \%)$ & $(7.1 \%)$ & $(10 \%)$ & $(6.4 \%)$ \\
\hline
\end{tabular}

Parmi ces courriels, $37779(92,1 \%)$ étaient hébergés par les grands prestataires de messagerie électronique, dont 31005 (75,6\%) chez les seuls Google, Microsoft, Orange et Yahoo (Tableau 2). Des courriels professionnels ou associés à un nom de domaine incluant l'identité du patient ont été fournis par $2878(7,0 \%)$ et $347(0,9 \%)$ patients, respectivement. Les patients ayant un courriel renseigné ont un suivi plus long $(72,4 \%$ versus $33,1 \%$ ayant un suivi de plus de trois mois) et étaient plus âgés (âge médian de 48 ans versus 45 ans).

\section{Tableau 2 : Description des adresses emails}

\begin{tabular}{|l|c|c|}
\hline & Effectifs & Localisation \\
\hline N & 41004 & \\
\hline Professionel & 2878 & \\
\hline Fournisseur, n (\%) & 347 & \\
\hline Google & $11730(28.6 \%)$ & USA \\
\hline Microsoft & $7802(19 \%)$ & USA \\
\hline Orange & $6402(15.6 \%)$ & Europe \\
\hline Yahoo & $5071(12.4 \%)$ & USA \\
\hline Free & $2427(5.9 \%)$ & Europe \\
\hline SFR & $1170(2.9 \%)$ & Europe \\
\hline La Poste & $815(2 \%)$ & Europe \\
\hline AOL & $330(0.8 \%)$ & USA \\
\hline NOOS & $319(0.8 \%)$ & Europe \\
Bouygues Telecom & $250(0.6 \%)$ & Europe \\
\hline
\end{tabular}

[1] Goyder C, Atherton H, Car M, Heneghan CJ, Car J. Email for clinical communication be tween healthcare professionals. Cochrane Database Syst Rev. 2015 Feb 20;(2):CD007979. doi 10.1002/14651858.CD007979.pub3.

[2] Newhouse N, Lupiáñez-Villanueva F, Codagnone C, Atherton H. Patient Use of Email for Health Care Communication Purposes Across 14 European Countries: An Analysis of Users According to Demographic and Health-Related Factors. J Med Internet Res [Internet] 2015 Mar 6;17(3):e58. doi: 10.2196/jmir.3700

Email : vincent.looten@aphp.fr 